\title{
Histological features of extratumoral breast lesions as a predictive factor of familial breast cancer
}

\author{
ANITA MANGIA $^{1}$, STEFANIA TOMMASI ${ }^{1}$, MICHELE BRUNO $^{1}$, ANDREA MALFETTONE ${ }^{1}$, \\ COSIMO D'AMICO ${ }^{2}$, FRANCESCO ALFREDO ZITO ${ }^{3}$, ANGELO PARADISO ${ }^{1}$ and GIOVANNI SIMONE ${ }^{3}$ \\ ${ }^{1}$ Clinical Experimental Oncology Laboratory, ${ }^{2}$ Histopathology Unit and \\ ${ }^{3}$ Woman Department, National Cancer Institute, 70126 Bari, Italy
}

Received July 8, 2009; Accepted October 19, 2009

DOI: $10.3892 /$ or_00000806

\begin{abstract}
The aim of this study was to verify whether histopathological features of extratumoral and of primary tumor breast tissue could play a role in identifying patients with familial characteristics. We examined the clinicopathological features of 504 patients with sporadic or familial breast cancer stratified for risk of BRCA mutation. Patients with a higher risk of being carrier of BRCA gene mutations were significantly associated with tumor poor differentiation $(\mathrm{p}=0.003)$, positive lymph node invasion $(\mathrm{p}=0.02)$ and presence of vascular peritumoral invasion $(\mathrm{p}=0.008)$. Among the extratumoral lesions, only the epithelial proliferative lesions were related to higher mutation risk both in the overall series and familial patients $(\mathrm{p}<0.0001$ and $\mathrm{p}=0.003$, respectively). Interestingly, a significant difference in terms of high mutation risk was observed in usual ductal hyperplasia lesions (UDH), $(p=0.002)$. We suggest that vascular peritumoral invasion and UDH lesions could predict a higher mutation risk of BRCA1 and BRCA2 genes and help in individuating patient candidates to further molecular analysis.
\end{abstract}

\section{Introduction}

Family history of breast cancer is a well established risk factor associated to mutations in high and low penetrance breast cancer susceptibility genes such as BRCA1, BRCA2, p53, ATM or PTEN but also to alterations not yet identified

Correspondence to: Dr Anita Mangia, Clinical Experimental Oncology Laboratory, National Cancer Institute, Via Hahnemann 10, 70126 Bari, Italy

E-mail: a.mangia@oncologico.bari.it

Abbreviations: UDH, usual ductal hyperplasia; DCIS, ductal carcinoma in situ; H\&E, haematoxylin and eosin; WHO, World Health Organization; IDC, infiltrating ductal carcinoma; ILC, infiltrating lobular carcinoma; ADH, atypical ductal hyperplasia; ER, estrogen receptor status; PgR, progesterone receptor status

Key words: extratumoral breast lesions, BRCA genes risk mutation, vascular peritumoral invasion, UDH
$(1,2)$. Furthermore, the study of Easton et al (3) demonstrated that common susceptibility loci can be reliably identified, and that they may together explain an appreciable fraction of the genetic variance in breast cancer risk. Consequently, there is a clear need for identification of phenotypic and molecular traits explaining the remaining familial cases of breast cancer.

Biological characteristics and clinical course of family history of breast cancer have been extensively reported (4-6), while the pathological characteristics of the surrounding nonmalignant tissue have been few investigated. Proliferative lesions in breast parenchyma include many histological changes and for some of them [ductal carcinoma in situ (DCIS), atypical hyperplasia and sclerosing adenosis] a potential interest for the progression of cancer has been demonstrated (7). Moreover, women with an autosomal dominant family history of breast cancer, independently from BRCA mutation, are prone to develop high-risk epithelial lesions (8).

Studies examining a possible association between presence of extratumoral lesions and presence of family history for breast cancer have been up to now performed in incomplete information on family history of disease (9), small series of hereditary breast cancer patients (10) and patients with basic information from only breast tissues obtained from prophylactic surgery (11).

The knowledge of histopathological characteristics of normal tissue where primary tumor of familial breast cancer patients is developed could be of particular interest. In fact, altered characteristics of the non-malignant surrounding tissue could represent the basis for genesis and progression of cancer disease in subjects with germline alterations and with hereditary susceptibility.

In the present study, we investigated the histopathological features of tumor and of surrounding tissue in patients with sporadic or familial breast cancer stratified for risk of BRCA mutation. Furthermore, we looked for a potential association between the normally considered 'breast cancer related risk factors' and the results of histological examination.

\section{Materials and methods}

Patients. We studied a retrospective series of 504 patients with a first diagnosis of breast cancer treated surgically in our Institute between February 2002 and March 2003 and all 
followed in genetic counseling program for familial breast/ ovarian cancer (12). Briefly, patients eligible for genetic counseling program were transferred to genetic counseling out-patients clinics where ad hoc teams updated their medical history and obtained informed consent to perform the molecular analyses. Patients were classified as having a family history of breast cancer according to criteria previously described (13). The risk of being a BRCA mutation carrier was calculated for each patient using the New Myriad II program (14). Patients were classified as having an 'increased risk' when the probability of finding a BRCA mutation was $\geq 10 \%$.

Histological examination. After surgical removal of biological tissues, the pathologist selected from the primary tumor and from surrounding macroscopically uninvolved breast tissues, samples destined for routine diagnostic practice at Pathology Department of our Institute. The extratumoral lesions were examined on the selected samples from the breast parenchyma at different distances from the primary nodule. Stored haematoxylin and eosin (H\&E) stained slides from each patient were evaluated by two pathologists (F.A.Z. and G.S.) who were unaware of the initial histological diagnoses and patient outcomes.

All tumors included in the study were classified according to the guidelines of the World Health Organization (WHO), (15) as infiltrating ductal carcinoma (IDC) not otherwise specified, infiltrating lobular carcinoma (ILC), DCIS and other carcinoma types (mixed tubular, tubular, medullary, atypical medullary, cribriform, mucinous, etc.).

Different histotypes, tumor size $(\leq 1.5 \mathrm{~cm},>1.5$ to $\leq 3 \mathrm{~cm}$, $>3 \mathrm{~cm}$ ), histological grading classified in 1, 2, 3 grade (performed according to the criteria described by Elston and Ellis) (16), axillary lymph node invasion (number of involved nodes in relation to total examined number), multifocality (absent, present), perineural invasion (absent, present), tumor necrosis (absent, present), calcification (absent, present), and vascular peritumoral invasion (absent, present) were recognised and reported. In H\&E-stained sections, vascular peritumoral invasion was considered evident if at least one tumor cell cluster was clearly visible in the vascular space with erytrocytes identified in the endothelial-lined space.

The histological classification of the benign extratumoral breast deseases was summarized into three categories: i) fibrocystic lesions (atrophy, fibrosis, fibrocystic changes); ii) epithelial proliferative lesions [usual ductal hyperplasia (UDH), ductal hyperplasia with columnar changes, flat epithelial atypia, atypical ductal hyperplasia (ADH), sclerosing adenosis and DCIS]; iii) benign tumors (fibroadenoma, papilloma).

Tumor proliferative activity (MIB-1 labeling index), estrogen $(\mathrm{ER})$ and progesterone $(\mathrm{PgR})$ receptor status were previously determined by immunohistochemistry $(13,17)$, and a cut-off of $10 \%$ of immunostained tumor cells was established for a tumor to be classified as negative or positive receptor and to differentiate between low or high proliferating tumors (13). All the analyses were performed in the Clinical Experimental Oncology Laboratory of the National Cancer Institute of Bari which participates in the INQUAT Quality Control programs (18) and is ISO 9001-2000 certified (DNV Certificate No. CERT-17885-2006-AQ-BRI-SINCERT).
Table I. Patients with sporadic breast cancer or with family history of breast and/or ovarian cancer.

No. of patients $(\%)$

\begin{tabular}{lrl}
\hline Overall series & 504 \\
Sporadic breast cancers & $336(66.7)$ \\
Family history & $168(33.3)$ \\
One first-degree relative, two or & $82(49)^{\mathrm{a}}$ \\
more second-degree relatives & & \\
One second-degree relative & $77(45.8)$ \\
Two or more first-degree relatives & 9 \\
\hline
\end{tabular}

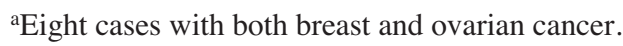

Statistical analysis. The association between clinicopathological features and risk of being carrier of BRCA gene mutations or presence of family history for breast cancer was assessed using the $\chi^{2}$ test. Differences were considered to be significant when p-value was $<0.05$. Statistical analyses were carried out with the SPSS statistical software (SPSS, Inc., Chicago, IL, USA).

\section{Results}

The characteristics of 504 patients are described in the Table I. Patients with familial breast cancer presented more frequently an ER-negative phenotype (34\%) with respect to sporadic patients $(23 \%),(\mathrm{p}=0.01)$. Moreover, MIB-1 positivity was significantly higher in sporadic cases $(73 \%)$ compared to familial cases $(62 \%),(\mathrm{p}=0.04)$.

Table II summarizes the risk of BRCA gene mutations in relation to clinicopathological features of all patients. The risk of being a BRCA gene mutation carrier is higher in patients aged between 35-50 years both considering the overall series $(\mathrm{p}<0.0001)$ and the familial cases $(\mathrm{p}=0.000)$. The patients with a higher risk showed to be significantly associated with higher histological grade ( $\mathrm{p}=0.003$ ), and positive lymph node invasion $(p=0.02)$. Similar result was also found when the analysis was restricted to the group of familial women: in fact, the 168 familial patients with a higher risk of BRCA genes mutation were significantly associated with histological grade and positive lymph node invasion ( $\mathrm{p}=0.047$ and $\mathrm{p}=0.008$, respectively). Moreover, the presence of vascular peritumoral invasion was significantly associated with a higher risk of BRCA gene mutations both in overall series and the familial subgroup ( $\mathrm{p}=0.008$ and $\mathrm{p}=0.048$, respectively).

When benign extratumoral lesions were examined (Table III), fibrocystic lesions were significantly associated with BRCA low mutation risk $(\mathrm{p}<0.0001)$ in all patients. In particular, atrophy lesion was specifically related to a low mutation risk ( $14 \%$ low risk vs. $0 \%$ high risk patients, $\mathrm{p}=0.0468$ ); and, atrophy and fibrosis were significantly present in sporadic patients with low risk gene mutation $(\mathrm{p}=0.04$, data not shown). Instead, when considering epithelial proliferative lesions in overall and familial series of patients, these were related to BRCA high mutation risk (58\% high 
Table II. Risk of BRCA gene mutations in relation to clinicopathological features.

\begin{tabular}{lcccc}
\hline & & \multicolumn{2}{l}{$\begin{array}{l}\text { Risk of BRCA } \\
\text { gene mutations }\end{array}$} & \\
\cline { 3 - 4 } Variables & All cases & Low & High & p-value \\
\hline & 504 & 459 & 45 &
\end{tabular}

$\begin{array}{lrrr}\text { Age (years) } & & & \\ \leq 35 & 21 & 13 & 8 \\ >35 \text { and }<50 & 142 & 115 & 27 \\ \geq 50 & 341 & 331 & 10\end{array}$

Histotype

$\begin{array}{lrrr}\text { IDC } & 408 & 372 & 36 \\ \text { ILC } & 26 & 23 & 3 \\ \text { DCIS } & 23 & 22 & 1 \\ \text { Others } & 47 & 42 & 5\end{array}$

Tumor size $(\mathrm{cm})$

$\leq 1.5$
$1.5<\mathrm{T} \leq 3$
$>3$

$\begin{array}{rrr}108 & 99 & 9 \\ 268 & 243 & 25 \\ 104 & 94 & 10 \\ & & \\ 78 & 75 & 3 \\ 227 & 212 & 15 \\ 141 & 119 & 22\end{array}$

Lymph node

invasion

Negative
Positive

Multifocality

Absent

Present

Perineural invasion

Absent

Present

Tumor necrosis

Absent

Present

Calcification

Absent

Present

Vascular peritumoral invasion

\begin{tabular}{lrrrr} 
Absent & 318 & 296 & 22 & \\
$\quad$ Present & 121 & 102 & 19 & 0.008 \\
Steroid receptors & & & & \\
$\quad \mathrm{ER}^{-}(\leq 10 \%)$ & 130 & 115 & 15 & NS \\
$\mathrm{ER}^{+}(>10 \%)$ & 359 & 29 & 30 & \\
$\operatorname{PgR}^{-}(\leq 10 \%)$ & 209 & 192 & 17 & $\mathrm{NS}$ \\
$\operatorname{PgR}^{+}(>10 \%)$ & 280 & 252 & 28 & \\
& & & & \\
MIB-1 & & & & \\
$\quad$ Negative $(\leq 10 \%)$ & 166 & 156 & 10 & NS \\
Positive $(>10 \%)$ & 323 & 289 & 34 & \\
\hline
\end{tabular}

NS, not significant $(p>0.05)$. risk vs. $26 \%$ low risk patients, $\mathrm{p}<0.0001$ and $45 \%$ high risk vs. $21 \%$ low risk patients, $\mathrm{p}=0.003$, respectively).

Among epithelial proliferative lesions, the higher mutation risk resulted significantly associated to UDH (42\% high risk vs. $17 \%$ low risk patients, $\mathrm{p}=0.002$ ), while a significant low risk is associated to ADH and sclerosing adenosis (44 vs. 24\%; $\mathrm{p}=0.0397 ; 19$ vs. $3 \%, \mathrm{p}=0.0273$, respectively). Any significant association was found analyzing singularly the presence of $\mathrm{UDH}, \mathrm{ADH}$ and sclerosing adenosis both in sporadic and in familial patients group. Benign tumors were all low risk patients and all of them had familial characteristics.

\section{Discussion}

NS In this study, we verified whether histopathological features of primary tumors and non-malignant surrounding breast tissue could be of some help in identifying risk familial patients. This information could be of great interest for several reasons: it could contribute to better understanding of the process of breast carcinogenesis and it could also represent a useful method to select patient candidates to further molecular studies.

We evaluated clinicopathological issues of 504 breast cancer patients with or without familiarity and with different risk of being a BRCA mutation carrier, focusing our attention on morphological features currently observed in clinical practice.

According to other studies (19), individual data on breast cancer in first and second-degree relatives of all the patients were collected, checked and analysed. We considered clinical features of familiarity to avoid exclusion of patients with hereditary breast cancer independent of BRCA, but dependent on other unknown genes.

The pathological features of BRCA associated cases are reported as being less favorable as compared to sporadic cases, with higher frequency of high grade (20), negative oestrogen and progesterone receptor (21) and rapidly proliferating tumors (22) as previously found (13). Moreover, Aaman et al (9) evidenced that the prevalence of proliferative breast diseases was slightly but not significantly lower in familial patients compared to sporadic. Differently from our study, Mohammed et al (20) and Molino et al (23) reported a significantly higher percentage of small tumors in women with family history.

In this study, familial cases resulted predominantly ER negative, while sporadic breast carcinomas showed higher proliferative activity. Furthermore, in agreement with Russo et al (24), we found no significant difference with other clinicopathological features.

When we analysed the relationship between the clinicopathological characteristics and risk of being a BRCA mutation carrier, the risk was lower in elderly patients as expected from other population-based studies. This is consistent with findings from other populations, indicating that the frequency of BRCA1 mutations is higher in early-onset breast cancer (25-27). According to Veronesi et al (28), higher histological grade and lymph node invasion were significantly more frequent in patients with high genetic risk. Moreover, we also found that patients with higher risk mutation proved to be significantly associated with the presence of vascular peri- 
Table III. Extratumoral breast lesions in relation to risk of BRCA genes mutation in 504 patients.

\begin{tabular}{|c|c|c|c|c|}
\hline \multirow[b]{2}{*}{ Type of parenchyma breast lesions } & \multirow[b]{2}{*}{ All patients } & \multicolumn{2}{|c|}{ Mutation risk } & \multirow[b]{2}{*}{ P-value } \\
\hline & & Low $(\%)$ & High $(\%)$ & \\
\hline Fibrocystic lesions & 339 & $315(70)$ & $24(42)$ & $<0.0001$ \\
\hline Atrophy & 45 & $45(14)$ & $0 \quad(0)$ & 0.0468 \\
\hline Fibrosis & 206 & 191 & 15 & NS \\
\hline Fibrocystic changes & 88 & 79 & 9 & NS \\
\hline Epithelial proliferative lesions & 151 & $118(26)$ & $33(58)$ & $<0.0001$ \\
\hline Usual ductal hyperplasia (UDH) & 34 & $20(17)$ & $14(42)$ & 0.0020 \\
\hline Ductal hyperplasia with columnar changes & 21 & 13 & 8 & NS \\
\hline Flat epythelial atypia & 7 & 6 & 1 & NS \\
\hline Atypical ductal hyperplasia (ADH) & 60 & $52(44)$ & $8(24)$ & 0.0397 \\
\hline Sclerosing adenosis & 23 & $22(19)$ & $1 \quad(3)$ & 0.0273 \\
\hline DCIS & 6 & 5 & 1 & NS \\
\hline Benign tumors & 14 & 14 & 0 & NS \\
\hline Fibroadenoma & 9 & 9 & 0 & NS \\
\hline Papillomas & 5 & 5 & 0 & NS \\
\hline
\end{tabular}

NS, not significant $(\mathrm{p}>0.05)$.

tumoral invasion that is an essential step in the metastatic cascade (29), and it has long been demonstrated that the detection of presence of tumor cells within vessels is a marker of a tumor with metastatic potential (30).

We further examined the role and the prevalence of benign proliferative lesions. No statistical difference was found when features of each extratumoral lesion in familial or sporadic breast cancer were compared. Moreover, examining the three histological categories of parenchyma breast lesions, the percentage of fibrocystic lesions, epithelial proliferative lesions and benign tumors were similar in patients with or without family history of breast cancer. Interestingly, also when the different criteria adopted to classify familiarity were considered, no difference was present regarding the type of parenchyma breast lesions (data not shown).

We found that the association of the fibrocystic lesions with low risk of mutation in all patients, and, in particular, of the atrophy with a lower mutation risk was due to the presence of atrophy and fibrosis in a higher number of low risk sporadic patients. Moreover, Worsham et al (31) demonstrated that the presence of fibrosis was protective against breast cancer, in fact women with fibrosis had a reduced risk for progression to breast cancer as compared with women without fibrosis.

In our study, the epithelial proliferative lesions of the overall series compared to fibrocystic lesions and benign tumors, were significantly associated with a high risk of mutation. This data was confirmed, also, analyzing mutation risk in familial patients.

$\mathrm{ADH}$ and sclerosing adenosis were significantly associated with a lower mutation risk. Our findings are in accord with those of Aaman et al (9) showing that patients with a family history of breast cancer had a slightly, but not significantly, decreased prevalence of ductal atypia (DCIS or
ADH) and sclerosing adenosis when compared with patients with no family history of cancer. Instead, the study of Webb et al (32) showed that women with proliferative benign breast diseases, in particular with atypia, were significantly associated with family history. Other studies also showed that women with a hereditary predisposition for breast cancer (11) and women with BRCA mutations (32) are prone to develop high risk lesions in their breasts, in particular lesions associated with an increased risk of invasive carcinoma (ADH, atypical lobular hyperplasia and lobular carcinoma in situ). The study of Hoogerbrugge et al (8) reported that women with familial breast cancer with a BRCA mutation had the highest predicted probability for the presence of high risk lesions in their breasts (ADH, DCIS). Moreover, Kroiss (6), in agreement with Hoogerbrugge et al (8) and Kauff et al (33), but differently from Adem et al (10), reported that BRCA gene mutation carriers had greater prevalence of premalignant lesions.

Several studies showed that the transformation of a benign breast lesion into an invasive carcinoma may occur more rapidly in BRCA carriers than in women suffering from breast cancer, perhaps because the environmental carcinogenesis is bypassed or accelerated in women with germline mutations in DNA repair genes. However, in our study, this hypothesis is contradicted by the high prevalence of epithelial proliferative lesions in high risk patients, in accord with Kroiss et al (6), and in particular by the high prevalence of UDH. In fact, we demonstrated that the UDH was significantly predominant in all patients with a higher risk of BRCA gene mutations, and indirectly confirmed by absence of a relationship between risk and fibrocystic lesions. These are more frequent in benign breast pathology, they do not normally include areas of ductal hyperplasia, or only in limited areas of the parenchyma. 
In conclusion, our data show that vascular peritumoral invasion could be a predictive factor of high risk, particularly, in familial patients with BRCA1 and BRCA2 genes mutation risk. Moreover, only the epithelial proliferative lesions, in particular UDH, are associated to a higher mutation risk patients. Intriguingly, UDH is a lesion widely observed both in sporadic and in familial breast cancer. Therefore, UDH should be considered as a part of the BRCA associated tumor spectrum and for this reason, we hypothesize that UDH could be the real target of malignant progression of this lesion. Our findings suggest that these women could be patient candidates to further molecular studies.

\section{Acknowledgements}

We would like to thank Mr. B. Stea for the statistical analysis, and Mrs. R. Daprile for her technical help in histology.

\section{References}

1. Ahmed M and Rahman M: ATM and breast cancer susceptibility. Oncogene 25: 5906-5911, 2006.

2. Antoniou AC and Easton DF: Models of genetic susceptibility to breast cancer. Oncogene 25: 5898-5905, 2006.

3. Easton DF, Pooley KA, Dunning AM, et al: Genome-wide association study identifies novel breast cancer susceptibility loci. Nature 447: 1087-1093, 2007.

4. Lakhani SR, Gusterson BA, Jacquemier J, et al: The pathology of familial breast cancer: pathological features of cancers in families not attributable to mutations in BRCA1 or BRCA2 Clin Cancer Res 6: 782-789, 2000

5. Palacios J, Honrado E, Osorio A, et al: Immunohistochemical characteristics defined by tissue microarray of hereditary breast cancer not attributable to BRCA1 or BRCA2 mutations: differences from breast carcinomas arising in BRCA1 and BRCA2 mutation carriers. Clin Cancer Res 9: 3606-3614, 2003.

6. Kroiss R, Winkler V, Kalteis K, et al: Prevalence of premalignant and malignant lesions in prophylactic mastectomy specimens of BRCA1 mutation carriers: comparison with a control group. J Cancer Res Clin Oncol 134: 1113-1121, 2008.

7. Hartmann LC, Sellers TA, Frost MH, et al: Benign breast disease and the risk of breast cancer. N Engl J Med 353: 229-237, 2005.

8. Hoogerbrugge N, Bult P, Bonenkamp JJ, et al: Numerous highrisk epithelial lesions in familial breast cancer. Eur J Cancer 42: 2492-2498, 2006.

9. Aaman TB, Stalsberg H and Thomas DB: Extra-tumoral breast tissue in breast-cancer patients: variations with a family history of breast cancer. WHO Collaborative Study of Neoplasia and Steroid Contraceptives. Int J Cancer 79: 39-43, 1998.

10. Adem C, Reynolds C, Soderberg CL, et al: Pathologic characteristics of breast parenchyma in patients with hereditary breast carcinoma, including BRCA1 and BRCA2 mutation carriers. Cancer 97: 1-11, 2003.

11. Hoogerbrugge N, Bult P, De Widt-Levert LM, et al: High prevalence of premalignant lesions in prophylactically removed breasts from women at hereditary risk for breast cancer. J Clin Oncol 21: 41-45, 2003.

12. Bruno M, Tommasi S, Stea B, et al: Awareness of breast cancer genetics and interest in predictive genetic testing: a survey in Southern Italian population. Ann Oncol (Suppl 1): I48-I54, 2004.

13. Tommasi S, Crapolicchio A, Lacalamita $\mathrm{R}$, et al: BRCA1 mutations and polymorphisms in a hospital-based consecutive series of breast cancer patients from Apulia, Italy. Mutat Res 578: 395-405, 2005 .
14. Frank TS, Deffenbaugh AM, Reid JE, et al: Clinical characteristics of individuals with germline mutations in BRCA1 and BRCA2: analysis of 10,000 individuals. J Clin Oncol 20: 1480-1490, 2002

15. Tavassoli FA and Devilee P: Pathology and genetics of tumors of the breast and female genital organs. In: The World Health Organization Classification of Tumors. Tavassoli FA and Devilee P (eds). IARC Press, Lyon, pp19-23, 2003.

16. Elston CW and Ellis IO: Pathological prognostic factors in breast cancer. I. The value of histological grade in breast cancer: experience from a large study with long-term follow-up. Histopathology 41: 154-161, 2002.

17. Mangia A, Chiriatti A, Tommasi S, et al: BRCA1, ER $\alpha$ expression and molecular BRCA1 alterations in familial breast cancer. Histol Histopathol 24: 69-76, 2009.

18. Paradiso A, Volpe S, Iacobacci A, et al: Quality control for biomarker determination in oncology: the experience of the Italian Network for Quality Assessment of Tumor Biomarker (INQAT). Int J Biol Markers 17: 201-214, 2002.

19. Collaborative Group on Hormonal Factors in Breast Cancer: Familial breast cancer: collaborative reanalysis of individual data from 52 epidemiological studies including 58,209 women with breast cancer and 101,986 women without the disease. Lancet 358: 1389-1399, 2001.

20. Mohammed SN, Smith P, Hodgson SV, et al: Family history and survival in premenopausal breast cancer. Br J Cancer 77 . 2252-2256, 1998.

21. Huang WY, Newman B, Millikan RC, Schell MJ, Hulka BS and Moorman PG: Hormone-related factors and risk of breast cancer in relation to estrogen receptor and progesterone receptor status. Am J Epidemiol 151: 703-714, 2000.

22. Chang $\mathrm{J}$ and Elledge RM: Clinical management of women with genomic BRCA1 and BRCA2 mutations. Breast Cancer Res Treat 69: 101-113, 2001.

23. Molino A, Giovannini M, Pedersini R, et al: Correlations between family history and cancer characteristics in 2256 breast cancer patients. Br J Cancer 91: 96-98, 2004.

24. Russo A, Herd-Smith A, Gestri D, Bianchi S, Vezzosi V, Rosselli Del Turco M and Cardona G: Does family history influence survival in breast cancer cases? Int J Cancer 99: 427-430, 2002 .

25. Eisinger F, Jacquemier J, Charpin C, et al: Mutations at BRCA1: the medullary breast carcinoma revisited. Cancer Res 58: 1588-1592, 1998.

26. Krainer M, Silva-Arrieta S, FitzGerald MG, et al: Differential contributions of BRCA1 and BRCA2 to early-onset breast cancer. N Engl J Med 15: 1416-1421, 1997.

27. Langston AA, Malone KE, Thompson JD, Daling JR and Ostrander EA: BRCA1 mutations in a population-based sample of young women with breast cancer. N Engl J Med 18: 137-142, 1996.

28. Veronesi A, De Giacomi C, Magri MD, et al: Familial breast cancer: characteristics and outcome of BRCA 1-2 positive and negative cases. BMC Cancer 5: 70, 2005

29. Pantel $\mathrm{K}$ and Brakenhoff RH: Dissecting the metastatic cascade. Nat Rev Cancer 4: 448-456, 2004.

30. Mohammed RA, Ellis IO, Lee AH and Martin SG: Vascular invasion in breast cancer; an overview of recent prognostic developments and molecular pathophysiological mechanisms (Review). Histopathology 55: 1-9, 2009.

31. Worsham MJ, Raju U, Lu M, Kapke A, Cheng J and Wolman SR: Multiplicity of benign breast lesions is a risk factor for progression to breast cancer. Clin Cancer Res 15: 5474-5479, 2007.

32. Webb PM, Byrne C, Schnitt SJ, et al: Family history of breast cancer, age and benign breast disease. Int J Cancer 100: 375-378, 2002.

33. Kauff ND, Brogi E, Scheuer L, et al: Epithelial lesions in prophylactic mastectomy specimens from women with BRCA mutations. Cancer 97: 1601-1608, 2003. 